\title{
Short Notes for Understanding the Basics of Nano Technology
}

\author{
D. Edison Selvaraj \\ S. Sivaa Priyan \\ Department of EEE \\ Panimalar Engineering College \\ Chennai, India \\ M. Rajmal Joshi \\ Department of EEE \\ Panimalar Engineering College \\ Chennai, India \\ S. Geethadevi \\ Department of EEE \\ Aurora Technological and \\ Research Institute, Uppal \\ Hyderabad, India
}

C. Pugazhendhi Sugumaran

Division of High Voltage

Engineering

College of Engineering

Guindy, Chennai, India
J. Ganesan
Department of EEE
Sree Sowdambika College of
Engineering, Aruppukottai, India

D. Krishnamoorthi
M. Raj Kumar

Department of EEE

Dhanalakshmi Srinivasan

College of Engineering and

Technology Chennai, India

\author{
S. Dinesh Kumar \\ Department of EEE \\ St. Peter's University, Avadi, \\ Chennai, India
}

\begin{abstract}
In this paper, the basic terms and definitions of nano technology was discussed. The characteristics, advantages and disadvantages of nano technology were discussed. Applications of nano technology were also mentioned. This paper would be useful to young engineers to study the fundamentals of nano particles. Two approaches used for nano technology were also elaborated.
\end{abstract}

Keywords: Nano Technology, Advantages, Disadvantages, Applications, Quantum dots, Nano particles, Nano wires

\section{INTRODUCTION}

\subsection{Definition of Nanotechnology}

The definition of nano technology was given by National Nanotechnology Initiative in U.S. Nano technology deals with structures having particle size of 1 to $100 \mathrm{~nm}$ [1-2]. Nanotechnology is the design, fabrication and use of nano structured systems and the growing, assembling of such systems mechanically, chemically or biologically to form nano scale systems and devices [3-4].

\subsection{Characteristics of nano technology}

1. It is very diverse.

2. It is based upon molecular self-assembly.

3. It is used in fields of science, organic chemistry, molecular biology, semiconductor physics, micro fabrication medicine, electronics \& energy production.

4. It was used to create many new materials.

5. It has impact on environment and economics.

6. It is the engineering of functional systems at the molecular scale.

7. It is used to make high performance products.

\subsection{Applications of nano technology}

1. Nano capacitors based filters

2. Nano transformer based SMPS

3. Nano cables

4. Nano insulators

5. Nano powders used for welding rods and electrodes

6. Nano based rectifiers

7. Nano resistance

8. Nano engineering materials

9. Silicon steel mixed with $5 \%$ nano magnetic properties

10. Nano sic Arrestors

11. Nano ZnO Arrestors

12. Nano technology used in receivers

13. Nano alloys

14. Nano capacitor based microphones

15. Nano electrets

16. Nano nuclear engineering

17. Nano thermo electric materials

18. Nano medicines

\subsection{Limitation of nano Technology}

1. Integration of nano structure and nano materials was not easy.

2. Demonstration of novel tools to study at nano meter was difficult. 
3. New measurement technologies were more challenging than ever.

4. It requires extremely sensitive instrumentation.

5. Monitoring and manipulation of the material processing in the atomic level was crucial.

6. Self-purification of nano materials makes doping very difficult.

7. Huge surface energy.

8. Uniform size distribution was difficult to achieve in nano materials.

9. It is not easy to achieve desired size, morphology, Chemical composition and physical properties.

10. Ostwald ripening and agglomeration would occur.

\subsection{Examples of nano particles}

1. $1 \mathrm{~nm}=10^{-9} \mathrm{~m}$

2. Spacing between the atoms in carbon-carbon bond was 0.12 to $0.15 \mathrm{~nm}$.

3. DNA double helix has a diameter of $2 \mathrm{~nm}$.

4. Bacteria have a length of $200 \mathrm{~nm}$.

\subsection{Quantum size effect}

The electronic properties of solids were altered with the reduction in particle size [5-6]. The physical, chemical, mechanical, electrical, thermal, magnetic \& optical properties of solids were changed with the decrease in the particle size. This was called as quantum size effect.

Example: 1.Opaque substance become transparent copper

2. Stable materials turn combustible aluminum

3. Insoluble materials become soluble gold

\section{MAIN APPROACHES IN NANO TECHNOLOGY}

There are two main approaches in nano technology.

1. Bottom up approach.

2. Top down approach.

\subsection{Bottom up approach}

1. In the bottom approach, materials and devices were built from molecular components which assemble themselves chemically by principles molecular recognition.

2. It utilities the concept of molecular self-assembly and supra molecule chemistry to automatically arrange themselves into some useful conformation

3. It involves the concept of molecular recognition.

4. Molecules can be designed due to non-covalent intermolecular forces

5. In this process, nano phase materials are produced by building of atom by atom, molecule by molecule or cluster by cluster. This process was used to build larger objects from smaller buildings blocks

6. It is opposite of top-down approach.

\subsubsection{Examples}

1. Watson-Crick base pairing

2. Enzyme - substrate interactions
3. Pulsed laser deposition

4. Chemical vapour deposition

5. Colloidal dispersion

6. Nano lithography

7. Nano manipulation

\subsubsection{Advantages}

1. Two or more components can be designed to be complementary and mutually attractive to make a complex.

2. It is used to produce devices in parallel.

3. It is cheaper than top-down method.

4. It is difficult for complex assembly.

5. It is used to obtain nano structures with fewer defects

6. More homogeneous chemical composition

7. Long life

8. Less time

9. Reduction of Gibbs free energy

10. Allows smaller geometries

11. Easier

12. Economical

13. It doesn't waste material

\subsubsection{Applications}

1. Production of salt \& nitrate

2. Growth of single crystal

3. Deposition of films

4. Fabrication of organic semiconductors

5. Manufacture of carbon nano tubes and silicon nano wires

\subsubsection{Disadvantages}

There was no difference in the physical properties of materials depending upon the synthesis method. But, chemical composition, crystallinity and microstructure of the material can change due to kinetic reasons depending upon the synthesis method. Consequently, the physical properties can also change.

\subsection{Top down approach}

In the top down approach, nano objects were constructed from larger entities without atomic level control.

\subsubsection{Examples}
1. Attrition or milling
2. Etching
3. Emulsification
4. Comminution

\subsubsection{Advantages}


1. Stronger covalent bonds were created by this method.

\subsubsection{Limitations}

1. Imperfection of surface structure

2. Crystallographic damage

3. Not smooth

4. Contains lot of impurities \& structural defects

5. Change in physical property \& surface chemistry due to large surface over volume ratio.

6. Reduced conductivity due to inelastic surface scattering

7. Excessive heat due to surface defects

8. In this process, bulk materials are broken into smaller nano size particles

9. Advantages

10. Stronger covalent bonds are created by this method.

\subsubsection{Applications}

1. Synthesis of nano particles such as $\mathrm{Al} 2 \mathrm{O} 3, \mathrm{SiO} 2, \mathrm{ZrO} 2$ nano fillers used in enamel

2. It is used in the synthesis of nano fillers such as $\mathrm{Al} 2 \mathrm{O} 3, \mathrm{SiO} 2, \mathrm{ZrO} 2, \mathrm{TiO} 2$ used in enamel.

\section{CLASSIFICATION OF NANO PARTICLES}

Nano particles were particles that would have dimension of $100 \mathrm{~nm}$ or less in size [7-8].

According to the shape of the crystallites nano materials were classified into four categories:

1. Clusters or powders $(\mathrm{MD}=0)$

2. Multi layers $(\mathrm{MD}=1)$

3. Ultrafine grained over layers or buried layers $(\mathrm{MD}=2)$

4. Nano materials composed of equiaxed nano meter sized grains $(\mathrm{MD}=3)$

Nano materials were classified according to their chemical composition as follows.

1. Materials with same chemical composition.

Example:

Semi crystalline polymers

Multi layers of thin film crystallites

separated by an amorphous layer

Si: N: H

$\mathrm{Nc}-\mathrm{si}$

2. Materials with different chemical

composition of grains.

Example:

Quantum well structures.
3. Materials having different chemical composition of its forming mater including different interfaces.

Example:

Ceramic of alumina with $\mathrm{Ga}$ in its interface

4. Nano materials formed by nano metre sized grains dispersed in a matrix of different chemical composition.

Example:

Precipitation hardened alloys.

Nano meter sized $\mathrm{Ni}-\mathrm{Al}$ precipitates

dispersed in a nickel matrix high temperature materials used in modern jet engines.

Precipitation hardened $\mathrm{Ni} 3 \mathrm{Al} / \mathrm{Ni}$ alloys.

\section{PROPERTIES OF NANO MATERIALS:}

The properties of many conventional materials change when formed from nano particles [9-11] This was due to the reason that nano particles have a greater surface area per weight than larger particles.

\subsection{Physical properties}

1. Large fraction of surface atoms.

2. Large surface energy.

3. Spatial confinement.

4. Reduced imperfections

5. Lower melting point.

6. Lower phase transition temperature.

7. Reduced lattice constants due to a huge fraction of surface atoms.

\subsection{Mechanical properties}

The enhancement in mechanical strength was simply due to the reduced probability of defects.

1. Carbon nanotubes were the strongest and stiffest materials. This was due to covalent sp2 bonds formed between the individual carbon atoms.

2. Multi walled CNT have a tensile strength of 63GPA.Individual CNT have a strength up to 100GPA.

3. CNT has a low density of 1.3 to $1.4 \mathrm{~g} / \mathrm{cm} 3$. Specific strength $48,000 \mathrm{kNmKg}-1$

4. CNT undergo bucking when placed under compressive, torsion or bending stress due to it hollow structure and high aspect ratio.

\subsection{Optical Properties}

Optical properties of nano material can be significantly different from bulk crystals [10]. The optical absorption peak of a semi conductor nano particle shifts to a short wavelength due to an 
increased band gap [12-13]. The optical absorption peak of metals was shifted by hundreds of $\mathrm{nm}$. The colour of metallic nano particle may change with their sizes due to surface Plasmon resonance.

\subsection{Electrical Properties}

1. The dielectric strength of the insulating materials were improved by adding nano fillers of $\mathrm{SiO} 2, \mathrm{TiO} 2, \mathrm{ZrO} 2, \mathrm{ZnO} 2$ and so an.

2. The insulation resistance of the enamel was increased by adding nano fillers of $\mathrm{SiO} 2, \mathrm{TiO} 2, \mathrm{ZrO} 2, \mathrm{ZnO}$ and so an.

3. Dielectric loss, temperature rise was increased in the enamel by the addition of $\mathrm{ZnO}$, Sic, $\mathrm{ZrO} 2, \mathrm{TiO} 2$, and $\mathrm{SiO} 2$ nano fillers.

4. The value of constant and capacitance was improved by the addition of nano fillers polyamide enamel used as the coating of the winding of the electrical machines.

5. The efficiency, thermal withstanding capacity of the electrical machines was improved by the addition of nano fillers to the enamel used for the coating of the windings of it.

6. Harmonics and EMI of the electrical machines were reduced by the addition of nano fillers to the enamel used for the coating of the windings of the electrical machines.

7. Electrical conductivity decreases with the reduction in dimension due to increased surface scattering particle charging energies were altered by hundreds of MV via particle size and safe. 8. Electrical conductivity of nano materials could be improved due to the better ordering in micro structure.

\subsection{Magnetic Properties}

Magnetic properties of nano materials were different from that of bulk materials. Ferromagnetism of bulk materials disappears and transfers to super paramagnetism in nano meter scale due to the huge surface energy. Iron oxide nano particles were used to improve MRI images of cancer tumours. The nano particles were coated with a peptide that binds to a cancer tumour. Once the nano particles were attached to the tumour, the magnetic property of the iron oxide enhances the images from the magnetic resonance imaginary scan.

\subsection{Thermal Properties}

Self purification was an intrinsic thermodynamic property of nano material Heat treatment increases the diffusion of impurities, intrinsic structural defects and dislocation. Increased perfection would have appreciable impact on the chemical and physical properties.

\subsection{Chemical Properties}

1. Chemical stability was improved.

2. Chemical properties were size dependent.

3. Chemical properties of nano materials were changed by adjusting the size, shape and extent of agglomeration.

\section{NANO PARTICLES}

Nano particles were particles that have particle size of $100 \mathrm{~nm}$ or less. The properties of many conventional materials change when formed from nano particles. This was due to the reason that nano particles have a greater surface area per weight than larger particles. Iron oxide nano particles were used to improve MRI images of cancer tumours. The nano particles were coated with a peptide that binds to a cancer tumour. Once the nano particles were attached to the tumour, the magnetic property of the iron oxide enhances the images from the magnetic resonance imagniary scan.

\section{QUANTUM DOTS}

1. A quantum dot was absorption of matter whose excitons were confined all three spatial dimensions.

2. They have electronic properties intermediate between bulk semiconductors and discrete molecules.

3. They were discovered at 1980 by Alexei E Kimov in a glass matrix and by Louis E. Brus in colloidal solutions.

4. The term "quantum dot" was coined by Mark Reed.

5. Quantum dots were observed in transistors, solar cells, LEDs and Diode lasers.

6. Quantum dots were stated as "Semiconductors whose electronic characteristic were closely related to the shape and size of the individual crystal."

\subsection{Characteristics of Quantum dots}

1. Smaller crystal size.

2. Larger band gap.

3. Greater difference in energy between the highest valence band and lowest band.

4. More energy was needed to excite the dot.

5. High level of control over the size of the crystals.

6. Very precise control over the conductive properties of the material.

7. Quantum dots of different sizes can be assembled into a gradient multi layer nano film.

8. Colour shift in fluorescent dye applications.

9. Diameter of quantum dots was $10 \mathrm{~nm}$.

\subsection{Production}

Quantum wires, wells and dots were produced by different methods.

1. Advanced Epitaxial technique 
(i) Molecular Beam epitaxy

(ii) Metal organic vapor phase epitaxy.

2. Chemical methods.

3. Ion implantation.

4. Lithography.

5. Colloidal synthesis.

6. Etching.

7. Quantum cryptography.

8. High temperature dual injection.

\subsection{Colloidal Synthesis}

Colloidal semiconductor nano crystals were synthesized from precursor compounds dissolved in solutions. The synthesis of colloidal quantum dots was based on a three component system composed of

1. Precursors

2. Organic surfactants.

3. Solvents.

The precursors transforms into monomers while heating a reaction medium to high temperature. Once the monomers reach a super saturation level, the nano crystal growth starts with a nucleation process. Temperature was an important factor in determining the optimal conditions for the nano crystal growth.

Another important factor was the monomer concentration.

The growth of nano crystals could occur in two regimes:

1. Focusing

2. Defocusing

\subsubsection{Focusing}

At higher monomer concentrations, the crystal size is small, resulting in the growth of all particles. In this regime, smaller particles grow faster than large ones resulting in focusing of the size distribution to yield mono disperse particles.

\subsubsection{Defocusing}

When the monomer concentration was depleted during growth the critical size becomes larger than the average size present and the distribution 'defocuses' as a result of Ostwald ripening.

Quantum dots were made of allow such as

1. Cadmium selenide.

2. Cadmium sulfide

3. Indium arsenide.

4. Indium phosphide.

5. Cadmium selenide sulfide.

\subsubsection{Advantages}

1. Convenient method

2. Used for commercial application.

3. Least toxic.

\subsection{Lithography and Etching}

Quantum dots obtained by lithography and etching has a dimension of $100 \mathrm{~nm}$. It was used for semi conductor hetero structures. A lateral quantum dot was obtained by electron beam lithography.

\subsection{Epitaxy}

6.5.1 Types

1. Molecular beam epitaxy.

2. Metal organic vapour phase epitaxy.

Material was grown on a substrate. This growth is called as stranski - krastanov to form quantum dot.

\subsubsection{Drawbacks}

1. Costly.

2. Lack of control.

\subsection{High Temperature Dual Injection}

It is impossible for the manufacture for large quantities of quantum dots.

\subsection{Types of quantum dots}

1. Core-shell structures.

2. Lateral quantum dots.

\subsubsection{Core-Shell structures}

Quantum dots having small regions of one material buried in another with a larger band gap were called as core-shell structures.

Example

1. CdSe in the core
2. $\quad \mathrm{ZnS}$ in the shell
3.

\subsubsection{Lateral Quantum Dots}

Individual quantum dots were created from two dimensional electron or hole gases present in remotely doped quantum wells or semi conductor hetro structures called as lateral quantum dots.

\subsection{Molecular Cluster Method}

1. Large quantities of high quality quantum dots were produced by using chemical precursors in the presence of a molecular cluster.

2. Individual molecules of the cluster would act as a seed or nucleation point.

3. High temperature nucleation was not necessary.

4. Highly scalable method.

\subsection{Assembly of quantum dots}

1. Viral assembly.

2. Electro chemical assembly.

\subsubsection{Viral Assembly}

Genetically engineered M13 bacteriophage viruses were used to create quantum dot bio composite structures. Genetically engineered viruses could recognize specific semiconductor surfaces through the method of selection by combinational phase display. The crystalline structures of wild type viruses such as Fd, M13 and TMV were adjusted by controlling

1. The solution concentrations.

2. Solution ionic strength. 
Advantages

3. External magnetic field applied to the solutions.

Self assembled quantum dots were obtained highly oriented and self supporting films were obtained from a phase and $\mathrm{ZnS}$ precursor solution. Genetic modification and selection were used to vary the length of bacteriophase and the type of inorganic material.

\subsubsection{Electrochemical Assembly}

Highly ordered arrays of quantum dots were obtained by electrochemical techniques.

\subsection{Confinement Potential}

Confinement in quantum dots could arise from electrostatic potential generated by

1. External electrodes.

2. Doping.

3. Strain.

4. Impurities.

The energy spectrum of the quantum was controlled by

1. Size.

2. Shape and

3. Strength of the confinement potential.

\subsection{Investigation}

Tunnelling spectroscopy was used for the investigation of quantum dots.

\subsection{Artificial Atoms}

The discrete transitions in the atomic spectra have resulted in the quantum dots called as artificial atoms.

\section{NANO WIRES}

Nano structures can be defined as structures that have a thickness or diameter constrained to tens of nano meters or less and an unconstrained length.

At this scale, quantum mechanical effects were important and hence they were called as quantum wires.

Types of nano wires:

1. Metallic nano wire

$$
\mathrm{Eg}: \mathrm{Ni}, \mathrm{pt}, \mathrm{Au} \text {. }
$$

2. Semi conducting nano wire

$\mathrm{Eg}: \mathrm{Si}, \mathrm{InP}, \mathrm{GaN}$.

3. Insulating Nano wire $\mathrm{Eg}: \mathrm{SiO} 2, \mathrm{TiO} 2$.

Molecular nano wires were of two types:

1. Organic nano wires

eg: DNA

2. Inorganic nano wires Eg: Mo6 S9 Ix.

\subsection{Properties}

1. It has aspect ratio of 1000 . Aspect ratio $=$ length/width.

2. They were called as one dimensional material.
3. Nano wires have interesting properties that were not seen in bulk or 3-D materials.

4. Electrons in nano wires were quantum confined laterally.

5. They occupy energy levels that were different from the traditional continuum of energy levels or bands found in bulk materials.

\subsection{Applications}

Nano wires were used to link tiny components into extremely small circuits.

\section{CONCLUSIONS}

In this paper, the basic terms and definitions of nano particles was discussed. The characteristics, advantages and disadvantages of nano technology were also discussed. Applications of nano particles were also mentioned. This paper would be useful to young engineers to study the fundamentals of nano technology. Quantum dots and nano wires were reviewed clearly.

\section{REFERENCES}

Selvaraj, D. Edison, et al. "Analysis of Efficiency, Thermal Withstanding Capacity and Electromagnetic Interference of Three Phase Squirrel Cage Induction Motor Coated with $\mathrm{SiO} 2 \& \mathrm{TiO} 2$ nano composite Filled Enamel." International Journal of Science and Engineering Applications 1.1 (2012): 17- 21.

[2] Edison Selvaraj, D., C. Pugazhendhi Sugumaran, and A. Sivaprakash."Characterization of Electrical and Thermal Properties of Enamel Filled with Carbon Nanotubes." Proceedings of the Third International Conference on Trends in Information, Telecommunication and Computing. Springer New York, 2013.

[3] Selvaraj, D. Edison. "Partial discharge characteristics of enamel filled with micro and nano composite of $\mathrm{siO} 2$ and TiO2." International Journal of Science and Engineering Applications 1.2 (2012): 95-101.

[4] Selvaraj, D. Edison. "Characterization of dielectric properties of the enamel filled with carbon nano tubes for the frequency range of $50 \mathrm{~Hz}-5 \mathrm{MHz}$ " International Journal of Science and Engineering Applications 1.2 (2012): 102-106.

[5] Selvaraj, D. Edison, and C. Pugazhendhi Sugumaran. "Comparative Analysis of Dielectric Properties of Enamel Filled with Various Nanofillers such as $\mathrm{ZrO} 2, \mathrm{Al} 2 \mathrm{O} 3$, CNT and ZnO." International Journal of Science and Engineering Applications 1.1 (2012): 51-55.

Babu, B. Gurukarthik, D. Edison Selvaraj, R. Srinivas, B. Guru Prakash, and R. Vishnu. "Analysis of Relative Permittivity and Tan Delta Characteristics of Silicone Rubber Based Nano-composites." International Journal of Scientific Engineering and Technology , pp.2201-206, 2012. 

$\mathrm{SiO} 2$ \& $\mathrm{TiO} 2$ Nano Composite Filled Enamel", International Journal of Engineering Sciences, Vol (2), No (4), 2013. pp. 115-118.

[8] Lieutenant Ganesan. J, Jeyadevi.S.Dr, and Edison Selvaraj. D, "Performance Analysis of Single Phase Induction Motor Coated with Al2O3 Nano Filler Mixed Enamel" ACEEE International Journal on Recent Trends in Engineering \& Technology Vol. 10, No. 1, Jan 2014.

[9] Edison Selvaraj. D, Pugazhendhi Sugumaran. C, Lieutenant Ganesan. J, Ramathilagam. J, "Analysis of Dielectric and Thermal Properties of Polyamide Enamel Filled with Carbon Nano tubes" International Journal of Nano science, Vol.12, Issue 3, June 2013.

[10] Lieutenant Ganesan. J, Edison Selvaraj. D, and Ramathilagam. J,"Experimental analysis of Thermal conductivity of enamel filled with micro and nano composite of $\mathrm{SiO} 2$ and $\mathrm{TiO} 2$ " International journal of Advanced Research in Electrical, Electronics and Instrumentation Engineering, Vol.2, Issue 7,pp. 2907-2912, 2013.

[11] Lieutenant Ganesan. J, Jeyadevi. S. Dr, and Edison Selvaraj. D, "Reduction of Electromagnetic Interference in Single Phase Induction Motor by coating the winding with $\mathrm{A} 12 \mathrm{O} 3$ nano filler mixed Enamel" International journal of Advanced Research in Electrical, Electronics and Instrumentation Engineering, Vol.2, Issue7,pp. 2913-2916, 2013.

[12] Lieutenant Ganesan. J, Edison Selvaraj. D, and Selva Kumar. B, "High Efficiency Induction Motor", International journal of Advanced Research in Electrical, Electronics and Instrumentation Engineering, Vol.2, Issue 2, pp. 750-754, 2013.

[13] Lieutenant Ganesan. J, Edison Selvaraj. D, GuruPrakash. B, Vishnu Prakash. R, Muthupandi. E, and BalaKumar. R, "Analysis of Efficiency and Thermal Withstanding Capacity of Single Phase Induction Motor Coated with Al2O3 Nano Filler Mixed Enamel", International journal of Advanced Research in Electrical, Electronics and Instrumentation Engineering, Vol.2,Issue 3,pp. 960-963, 2013. 